\title{
The efficiency of the in vitro osteo/dentinogenic differentiation of human dental pulp cells, periodontal ligament cells and gingival fibroblasts
}

\author{
JUNG-KWON CHOI, HYO-IN HWANG and YOUNG-JOO JANG \\ Laboratory of Cell Cycle and Signal Transduction, Department of Nanobiomedical Science \\ and BK21 PLUS NBM Global Research Center for Regenerative Medicine, Dankook University, \\ Cheonan-si, Chungnam 330-714, Republic of Korea
}

Received April 2, 2014; Accepted October 20, 2014

DOI: $10.3892 /$ ijmm.2014.1986

\begin{abstract}
Although the primary cell cultures from dental pulp and other oral tissue are frequently used to study osteogenic potential and stem cell responses, few systematic and comparative studies on stemness for the dentinogenic differentiation of these cells have been conducted. In the present study, to investigate the stemness of oral primary cells during extended culture, human adult dental pulp cells (hDPCs), periodontal ligament stem cells (hPDLSCs) and gingival fibroblasts (hGFs) were obtained and cultured from pulp tissue, periodontal ligaments, and marginal and attached gingival tissue of extracted third molars, respectively. As shown by fluorescence-activated cell sorting analysis and immunophenotyping, the mesenchymal stem cell markers, CD44, CD73, CD90, CD146 and CD166, were highly expressed in early passage hDPCs, hPDLSCs and hGFs. However, when the cells were treated with osteogenic additives, mineralization markedly increased in the hDPCs and hPDLSCs, but not in the hGFs. Moreover, the expression of dentinogenic markers, such as dentin sialophosphoprotein and dentin matrix protein-1, appeared to decrease during extended culture past passage number 8 of the hDPCs and hPDLSCs. These data suggest that hDPCs and hPDLSCs may have differentiation potential during the early passages, and that their progenitor potential is diminished during extended culture. The hGFs did not show differentiation capability during culture, even though they contained general mesenchymal stem cell surface proteins. The transcriptional expression of dentinogenic markers in hDPCs was not affected by co-culture with hPDLSCs and/or hGFs.
\end{abstract}

Correspondence to: Professor Young-Joo Jang, Laboratory of Cell Cycle and Signal Transduction, Department of Nanobiomedical Science and BK21 PLUS NBM Global Research Center for Regenerative Medicine, Dankook University, 29 Anseo-Dong, Cheonan-Si, Chungnam 330-714, Republic of Korea

E-mail: yjjang@dankook.ac.kr

Key words: human adult dental pulp cells, periodontal ligament cells, gingival fibroblasts, dentinogenic differentiation, co-culture

\section{Introduction}

Tissue-specific stem cells have differentiation potential and regenerate missing tissue continuously $(1,2)$. At present, tissue regeneration using stem cells is considered effective with good therapeutic value. However, there are some technical issues, such as where stem cells can be obtained from, how to induce differentiation into target tissue cells and how long the cells can maintain their healthy status in the body. Adult stem cells, such as hematopoietic, epithelial and neural stem cells, are found in some parts of the human body (3-6). Dental pulp cells (DPCs), which have the capacity to produce dentin matrix, can be differentiated into osteoblasts, chondrocytes, adipocytes, endothelial cells and neurons (7-10). The undifferentiated progenitor pulp cells, residing in the perivascular niche of the dental pulp, the so-called subodontoblastic cell-rich zone, may migrate to the site of injury for reparative dentinogenesis (11-13). Human dental pulp contains a stromal stem cell population with high proliferative potential that induces mineralized dentinogenesis and is positive for cell surface markers, such as STRO-1, CD44 and CD146 (14-16). Although the expression levels of these factors differ among species, including pigs, rats and rodents, some markers provide the possibility of detecting specific stemness in human DPCs (hDPCs) (17-20). In addition to DPCs, human periodontal ligament stem cells (hPDLSCs) are found near the periodontal ligament of the teeth and are involved in the regeneration of the periodontal ligament, alveolar bone and cementum $(12,21-23)$. The morphology and mineralization of hPDLSCs isolated from periodontal ligament tissue is similar to that of hDPCs $(21,24)$. Gingival fibroblasts (GFs) organize the oral tissue attached to the alveolar bone of tooth sockets and function as a biological mucosal barrier, producing inflammatory cytokines such as interleukin (IL)-6 and IL-8 (25). Mesenchymal cells from the oral mucosa contain stem cell factors that are excreted from human GF (hGF)-like cells $(26,27)$. Although it has been previously reported that GFs contribute to oral wound healing with phenotypically different features from other fibroblasts and contain a mesenchymal stem cell population (28), their stemness remains unclear. The present study focused on a comparison of the in vitro osteo/dentinogenic differentiation potential of hPDLSCs, hGFs and hDPCs 
together during extended culture. In addition, we investigated whether hPDLSCs and/or hGFs affect the osteo/dentinogenic differentiation of hDPCs in a co-culture experiment.

\section{Materials and methods}

Isolation and culture of $h D P C s, h P D L S C$ s and $h G F s$. Five human third molars were collected from patients aged 20-25 years under guidelines approved by the Institutional Review Board (IRB) of Dankook Dental Hospital, Cheonan-si, Korea. Following extraction, the periodontal ligament and attached gingival tissue were scraped from the surface of the teeth. The dental crown was fractured and the dental pulp was recovered. The tissue was chopped with a scalpel into small sections under sterilized conditions, and the suspension was incubated in $\alpha$-MEM (Gibco/ Life Technologies, Carslbad, CA, USA) containing 20\% fetal bovine serum (FBS; HyClone, Logan, UT, USA) and antibiotics at $37^{\circ} \mathrm{C}$ in a humid atmosphere containing $5 \% \mathrm{CO}_{2}$ for 3-5 days. The cells stretched out from the tissue sections were re-plated, and $80 \%$ confluent cultures were used as passage number 0 . Adherent cells grown to $70 \%$ confluency were subcultured at a 1/5 dilution for later passage. The media were replaced every 3 days until the cells had grown to the appropriate confluency. The primary cultures of pulp, periodontal ligament and gingival tissue were performed separately with the cells of each tooth, and each culture was analyzed separately. For differentiation, $5 \mathrm{mM} \beta$-glycerophosphate, $100 \mathrm{nM}$ dexamethasone and $100 \mu \mathrm{M}$ ascorbic acid were added to the culture media as additives and the cells were incubated for 8-14 days, as previously described (15). For the indirect co-culture of the cells, $2 \times 10^{5}$ cells of human DPCs at passage number 2 were cultured in a 6-well plate, and the same numbers of hPDLSCs and hGFs were seeded into a $40-\mu \mathrm{m}$ cell strainer (BD Falcon ${ }^{\mathrm{TM}}$; BD Biosciences, San Jose, CA, USA), was placed on a 6-well plate containing pulp cells for indirect co-culture. For culture on polycaprolactone (PCL) membrane (kind gift from Dr H.W. Kim, Dankook University), the membranes were sterilized in $70 \%$ alcohol overnight, rinsed thoroughly with PBS, and treated with ultraviolet (UV) light overnight. After sterilization, PCL membranes were placed in tissue culture plates. The cell suspension at a density of 80,000 cells $/ \mathrm{ml}$ was seeded into each well and maintained in a humidified atmosphere with $5 \% \mathrm{CO}_{2}$ at $37^{\circ} \mathrm{C}$.

Flow cytometry and immunophenotyping. At each passage, the hDPCs, hPDLs and hGFs were harvested using trypsin-free dissociation buffer (Millipore, Billerica, MA, USA) and suspended in PBS containing 5\% fetal bovine serum at $1 \times 10^{6}$ cells $/ \mathrm{ml}$ of concentration. To immunophenotype the primary cells, the cells were treated with antibodies against the following human cell surface antigens: CD24 (555428), CD44 (550989), CD73 (550257), CD90 (555596), CD106 (555647), CD146 (550315) and CD166 (559263). Anti-human IgG was used as a negative control. All the antibodies were labeled with phycoerythrin (PE) and purchased from BD Biosciences. PE-conjugated STRO-1 antibody was purchased from Santa Cruz Biotechnology (sc-47733; Santa Cruz, CA, USA). The cells were analyzed by flow cytometry using a FACSCalibur flow cytometer (BD Biosciences). The FACS data were analyzed and plotted by measuring the mean fluorescence using CellQuest software and the WinMDI program.
Alizarin red $S$ staining. The hDPCs, hPDLs and hGFs were cultured and stimulated for differentiation as previously described (15). Culture dishes were washed with $\mathrm{Ca}^{2+}$-free PBS twice. The cells were fixed with $70 \%$ ethanol for $5 \mathrm{~min}$ at room temperature, and were then allowed to dry completely. For staining, the cells were treated with $2 \%$ Alizarin red S (pH 4.5) (Sigma-Aldrich, St. Louis, MO, USA) for $1 \mathrm{~min}$ and then washed with distilled water. For quantification, the deposits bound to Alizarin red were extracted with $10 \%$ acetic acid. Following neutralization with $10 \%$ ammonium hydroxide and the optical density was analyzed at a wavelength of $405 \mathrm{~nm}$.

Western blot analysis. Total protein was extracted from the cells using cell lysis buffer (10 mM Tris, pH 7.5, $150 \mathrm{mM} \mathrm{NaCl}$, $1 \mathrm{mM}$ EDTA and $0.5 \%$ NP-40), and the protein concentration was estimated using Bradford reagent (Bio-Rad Laboratories, Hercules, MA, USA). Forty micrograms of total protein were resolved on SDS-PAGE, electrotransferred onto a PVDF membrane, and probed with the appropriate antibodies in TBS containing $0.05 \%$ Tween-20 and 5\% skim milk. Protein signals were visualized using an enhanced chemiluminescence (ECL) detection system (Amersham Biosciences, Piscataway, NJ, USA). Antibodies for dentin sialophosphoprotein (DSPP; SC-73633) and dentin matrix protein-1 (DMP-1; M176) were obtained from Santa Cruz Biotechnology and Takara Bio Inc. (Tokyo, Japan), respectively. The primary antibodies and secondary antibody, goat anti-rabbit IgG (Millipore), were used at a dilution of 1:200-1:500 and 1:2,000, respectively.

Reverse transcription quantitative (real-time) polymerase chain reaction $(R T-q P C R)$. Total RNA was isolated from $5 \times 10^{5}-1 \times 10^{6}$ cells using the NucleoSpin ${ }^{\circledR}$ RNA XS RNA prep kit (Macherey-Nagel Inc., Bethlehem, PA, USA) and $2 \mu \mathrm{g}$ of total RNA were reverse-transcribed into cDNA using the iScript cDNA Synthesis kit (Bio-Rad Laboratories) with oligo primer in $25 \mu \mathrm{l}$ of reaction mixture consisting of RNA, dNTP, primer, RNasin and AMV reverse transcriptase. Following a denaturation step, synthesis reaction was performed for $60 \mathrm{~min}$ at $42^{\circ} \mathrm{C}$. Equal amounts of cDNA were used for the real-time amplification of the target genes in triplication using the SsoFast EvaGreen Supermix/iQ SYBR ${ }^{\circledR}$-Green Supermix kit (Bio-Rad Laboratories) according to the instructions for the CFX96 Real-Time PCR detection system (Bio-Rad Laboratories). The relative expression levels of GAPDH, DMP-1 and DSPP were determined. The primers used for realtime PCR were as follows: GAPDH, forward, 5'-GGAGTCCACTGGCGTCTTCAC-3' and reverse, 5'-GCTG ATGATCTTGAGGCTGTTGTC-3'; DMP-1, forward, 5'-CAG GAAGAGGTGGTGAGTGAGTC-3' and reverse, 5'-CTGGA TTCGCTGTCTGCTTGC-3'; DSPP, forward, 5'-CAGTAC AGGATGAGTTAAATGCCAGTG-3' and reverse, 5'-CCATT CCCTTCTCCCTTGTGACC-3'. The expression of GAPDH was used as a reference. CFX Manager ${ }^{\mathrm{TM}}$ software version 1.5 was used in the analysis of the results.

\section{Results}

hDPC, $h P D L S C$ and hGF primary cultures contain stem cell populations. To investigate passage-dependence of stemness from the oral cell population, we obtained pulp, periodontal 

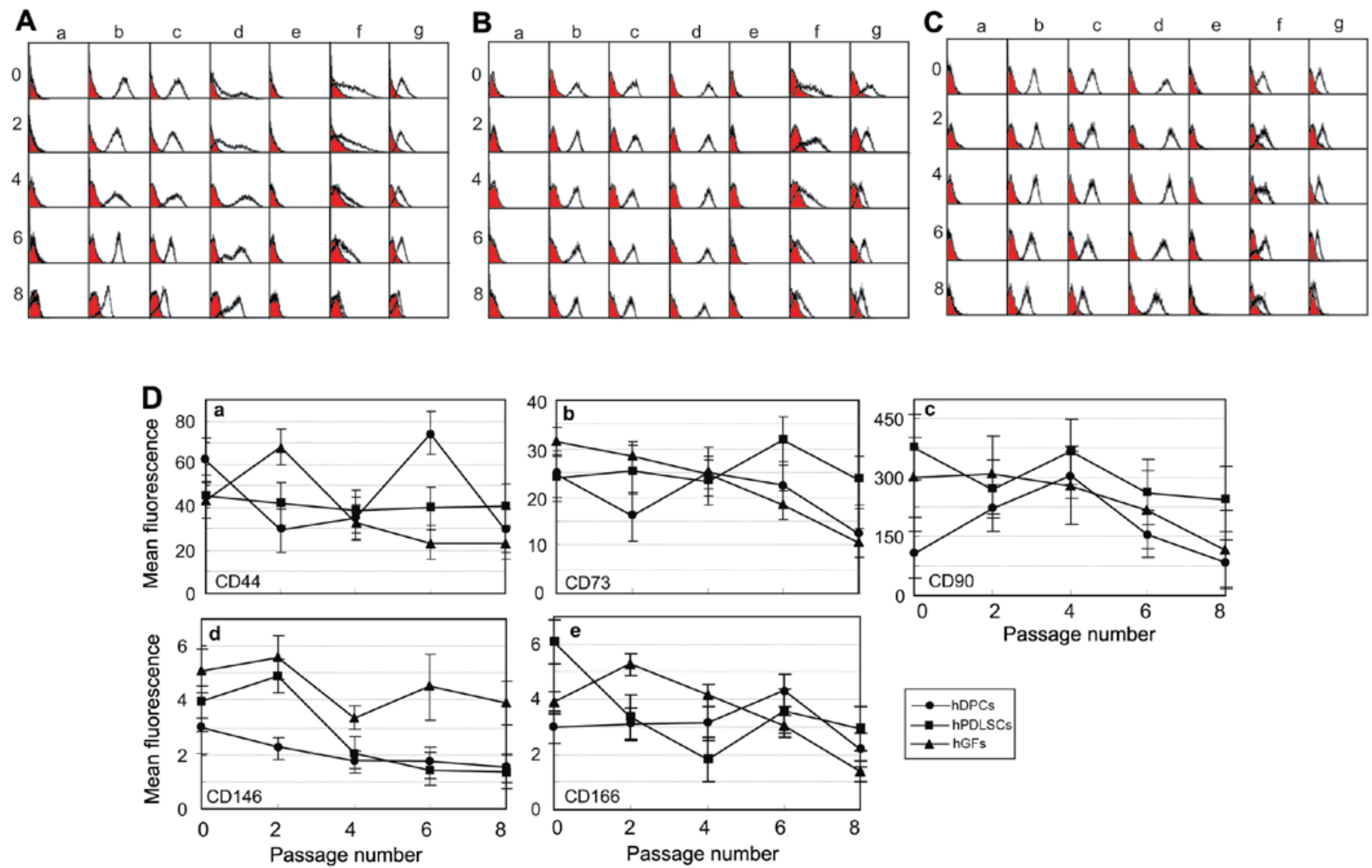

-hGFs

Figure 1. Immunophenotyping of (A) human dental pulp cells (hDPCs), (B) periodontal ligament stem cells (hPDLSCs) and (C) gingival fibroblasts (hGFs) Cells were treated with phycoerythrin (PE)-conjugated antibodies against the cell surface antigens. Anti-human IgG was used as a negative control (filled curves), and the fluorescence intensity of the specific antibody binding is indicated by open curves. Cells at passages 0-8 are shown. Panels a, CD24; b, CD44; c, CD73; d, CD90; e, CD106; f, CD146; and g, CD166. (D) The antibody binding affinity was quantified and re-estimated as the mean fluorescence intensity using CellQuest software and the WinMDI 2.9 program. panels a, CD44; b, CD73; c, CD90; d, CD146; and e, CD166.

ligament and attached gingival tissue from human adult third molars, and performed primary cultures. Due to the fact that specific hDPSC and hPDLSC surface markers have not been identified to date, general mesenchymal stem cell markers were used to detect the stem cell population within the primary cultures. We used CD44, CD73, CD90, CD106, CD146, CD166 and STRO-1 to identify the stem cell populations. CD44, CD73, CD90, CD146 and STRO-1 are general surface proteins for identifying mesenchymal and hematopoietic stem cells (29-36). In addition, both CD106 (VCAM-1) and CD166 are markers for bone marrow mesenchymal stem cells $(37,38)$. The cells from each primary culture were pooled and treated with surface antibody conjugated with PE, and the fluorescence intensity of the cells was analyzed by flow cytometry. The hDPCs and hPDLSCs during early passage strongly expressed CD44, CD73, CD90, CD146 and CD166 (Fig. 1A and B, panels b-d, f and g). Although CD24 and CD106 are dental apical papilla stem cell markers $(9,39)$, they were not expressed in the hDPCs and hPDLSCs used in the present study (Fig. 1A and B, panels a and e). In addition, STRO-1 was not a proper surface marker to define dental stem cells from pulp and the periodontal ligament (data not shown), although it has been used as a general mesenchymal stem cell marker. The expression of CD44, CD73, CD90 and CD166 increased during hDPC passage numbers 4-6 and decreased at passage number 8 (Fig. 1D, circle in panels a-c and e). CD146 expression increased during early passage (Fig. 1D, circle in panel d). The levels of CD146 and CD166 peaked during the initial passages of hPDLSCs (Fig. 1D, square in panels d and e), whereas CD73 and CD90 were highly expressed during passage numbers 4-6 (Fig. 1D, square in panels $b$ and c). The expression of CD44 in the hPDLSCs was consistent during the extended culture (Fig. 1D, square in panel a). When the cells from the attached gingival tissue were cultured, the very early passage numbers expressed the general mesenchymal stem cell markers, CD44, CD73, CD90, CD146 and CD166 [Fig. 1C and $\mathrm{D}$ (triangle in panels a-e)]. The expression of these markers decreased rapidly with the increase in passage number during extended culture. These findings indicate that hGFs, as well as hDPCs and hPDLSCs, contain a stem cell population during early passage in primary culture.

Mineralization efficiency of $h D P C s, h P D L S C s$ and $h G F s$. When we cultured hDPCs, hPDLSCs and hGFs devided from tissue sections of third molar teeth, cells from individual cultures had a fibroblast-like, spindle-shaped and elongated morphology without treatment with differentiation additives (Fig. 2 ,panel 1). To investigate the osteo/dentinogenic potential of these dental cells, cells from passage number 2 were treated with differentiation additives containing $\beta$-glycerophosphate, ascorbic acid and 


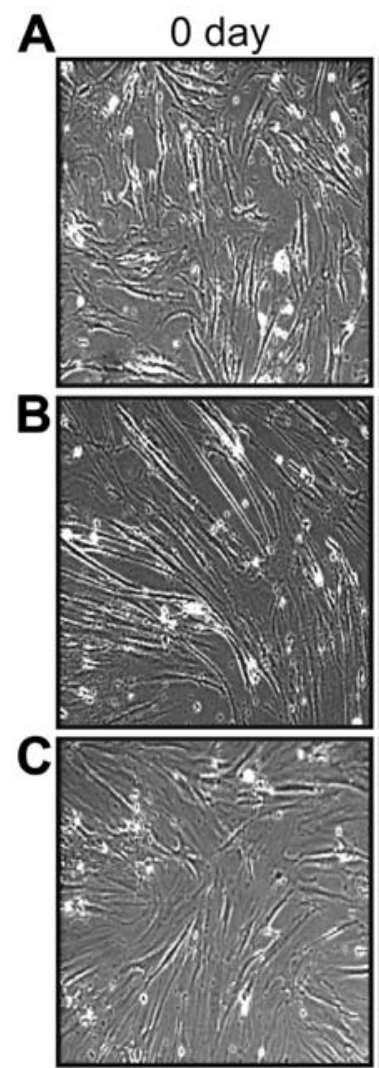

1
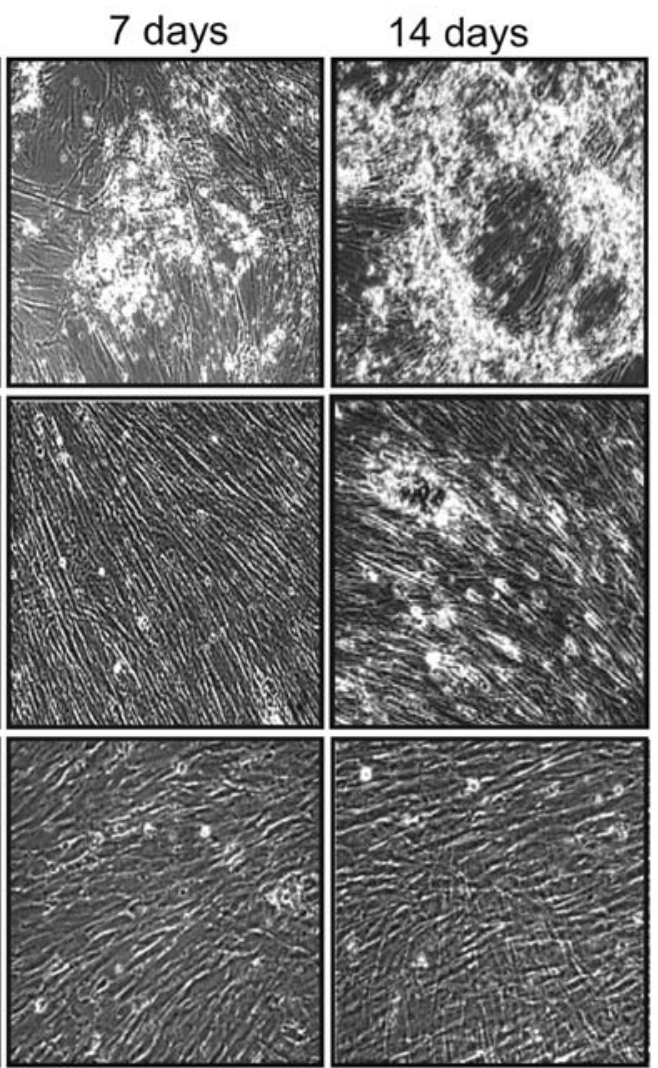

2

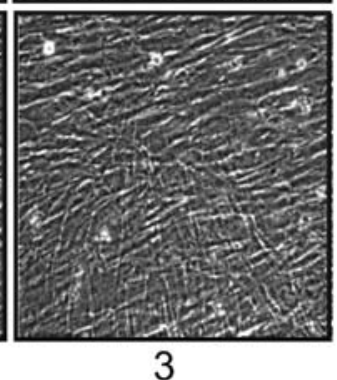

Figure 2. Phenotypes of cells before and after treatment with osteogenic medium. (A) Human adult dental pulp cells, (B) periodontal ligament stem cells and (C) gingival fibroblasts from passage number 2 during extended culture were treated with $5 \mathrm{mM} \beta$-glycerophosphate, $100 \mu \mathrm{M}$ ascorbic acid, and $100 \mathrm{nM}$ dexamethasone to induce differentiation. Folloiwng incubation for the indicated periods of time, cell morphology and mineralization were observed. panel 1 , before treatment with additives; panel 2, incubation for 7 days after treatment with additives; and panel 3, incubation for 14 days after treatment with additives.

dexamethasone. After 7 days of treatment with differentiation additives, the hDPCs and hPDLSCs formed sporadic aggregates (Fig. 2Aand B, panel 2). These aggregates developed into a layer covering the cell surface after 2 weeks (Fig. 2A and B, panel 3). During the induction of differentiation, the depository layer produced on the hDPCs was more extensive than that on the hPDLSCs and covered the entire adherent layer on the surface of the hDPCs. Although these aggregative structures were also formed without additive treatment during early passage in the hDPCs and hPDLSCs, the efficiency of aggregate formation was very low (data not shown), as shown in a previous study (15). The hGFs also showed a fibroblast-like and elongated morphology without treatment with differentiation additives, similar to the hDPCs and hPDLSCs (Fig. 2C, panel 1). However, following the induction of differentiation, the population of hGFs appeared as a fine mesh-like structure without the depository layer overspreading the entire culture (Fig. 2C, panel 3). To investigate whether these aggregates or the sheath-like structure included calcium deposits by mineralization, the cells were stained with Alizarin red S solution. Alizarin red S-positive deposits were observed over the whole cell layer evenly in both the hDPCs and hPDLSCs from early passage (Fig. 3A, panel 2 parts a and c), whereas these deposits were not detected in the hGFs (Fig. 3A, panel 2 part b). The hDPCs and hPDLSCs in extended passage did not form calcium deposits under differentiation conditions (Fig. 3B, panel 2 parts a and c). Due to the fact that the efficiency of cell adhesion and osteo/dentinogenic differentiation of hDPCs, hPDLSCs and hGFs may be affected on biomaterial surface mimicking the mechanical environment of the extracellular matrix, these cells were cultured on poly( $\varepsilon$-caprolactone) (PCL) fibrous membranes, as previously described $(40,41)$. After 2 weeks of differentiation, the amount of mineral deposits from the hDPCs cultured on the PCL membranes was greater than that from the hPDLSCs and hGFs (Fig. 3C, parts a and b, lanes 1-3). Under the same conditions, the hGFs did not form mineral deposits during culture on biomaterial (Fig. 3C, parts a and b, lane 2).

Expression of dentin markers in hDPCs, hPDLSCs and $h G F s$ under differentiation conditions. To investigate the dentinogenic potential of hDPCs, hPDLSCs and hGFs during differentiation, the endogenous protein levels of dentinogenic markers were analyzed during extended cell culture. Under differentiation conditions, the expression of DSPP in the hDPCs and hPDLSCs markedly increased (Fig. 4A and B, $\alpha$-DSPP in panel b). The expression levels of DMP-1 also increased in both cell types under the same conditions, although at lower levels than those of DSPP (Fig. 4A and B, $\alpha$-DMP in panel b). Of note, dentin marker expression decreased with the increasing passage number during extended culture. In both cases, dentin marker expression peaked during early passage (passage numbers 4-6), and diminished at passage 8 (Fig. 4A and B, 

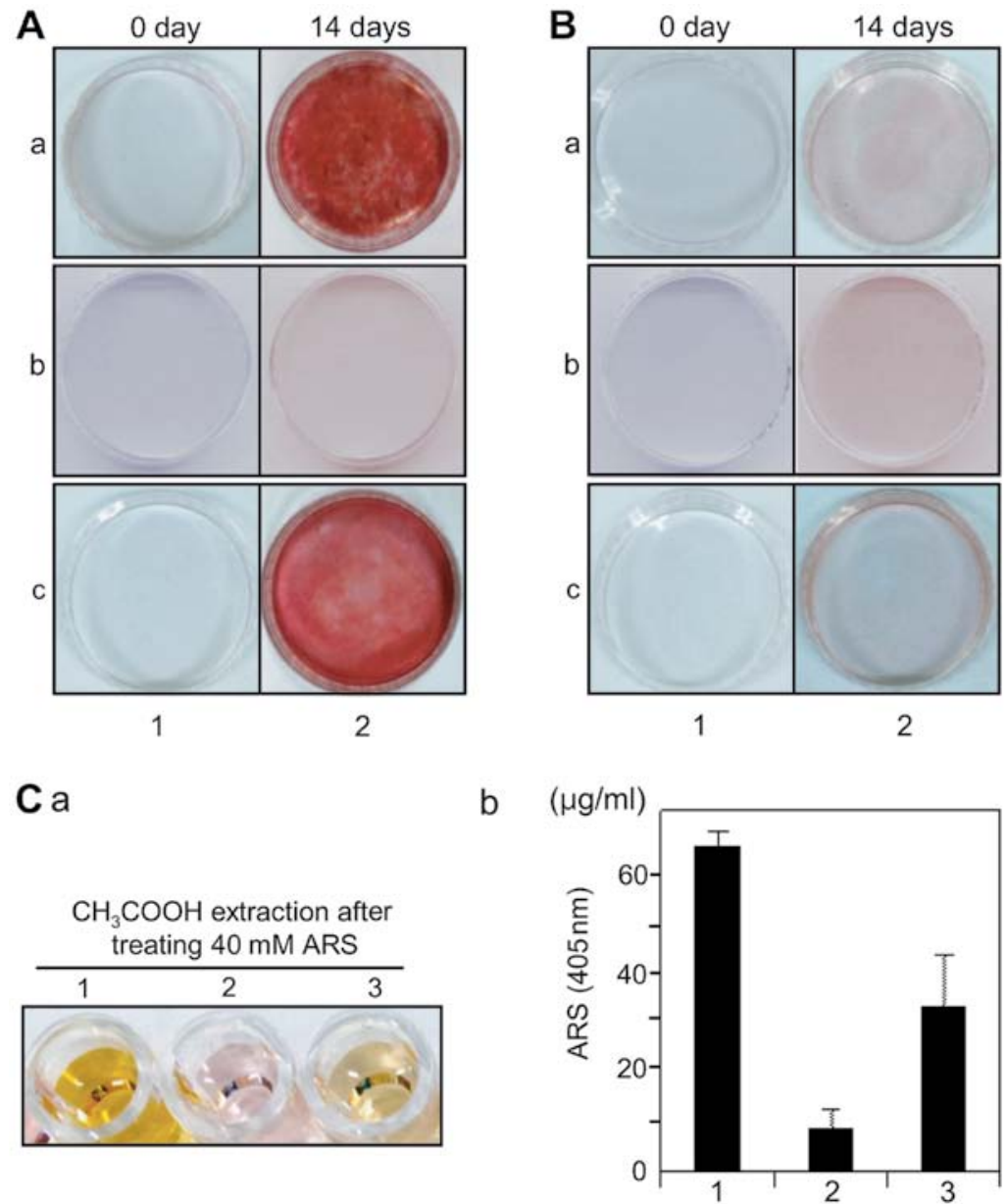

Figure 3. Mineralization of dental pulp cells, periodontal ligament cells, and gingival fibroblasts under differentiation conditions. Cells from (A) passage number 2 and (B) passage number 13 were treated without (panel 1) or with (panel 2) additives for differentiation. a, Human adult dental pulp cells; b, gingival fibroblasts; c, periodontal ligament stem cells. (C) Quantification of mineralization. The Alizarin red S (ARS)-mineral complex was (a) extracted and its concentration was measured by (b) absorbance at $405 \mathrm{~nm}$. Lane 1, Human adult dental pulp cells; lane 2, gingival fibroblast; and lane 3, periodontal ligament cells.
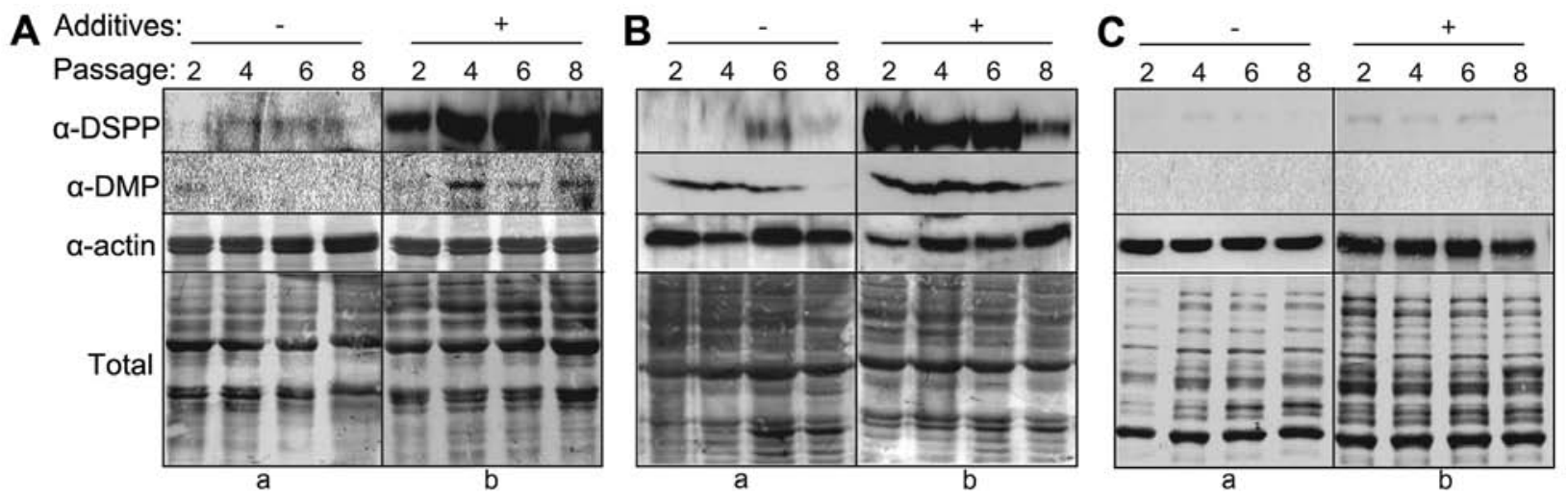

Figure 4. Protein expression of dentinogenic markers representing differentiation during extended culture. (A) Human adult dental pulp cells, (B) periodontal ligament stem cells, and (C) gingival fibroblasts were collected at the indicated passage number from culture. The expression levels of dentin sialophosphoprotein (DSPP) and dentin matrix protein 1 (DMP-1) were analyzed by western blot analysis using specific antibodies ( $\alpha$-DSPP and $\alpha$-DMP). The endogenous actin and total protein used in western blot analysis are shown in the lower panels ( $\alpha$-actin and total). (A-C) For differentiation, cells were treated with the additives for 14 days: -, without treatment (panel a); +, treatment with differentiation additives (panel b).

lane 8). By contrast, dentin marker expression did not increase in the hGFs during extended culture (Fig. 4C, $\alpha$-DSPP and $\alpha$-DMP in panel b).
Dentinogenic potential of hDPCs during co-culture with hPDLSCs and/or hGFs. We performed an indirect co-culture experiment with the hPDLSCs and/or hGFs to determine 

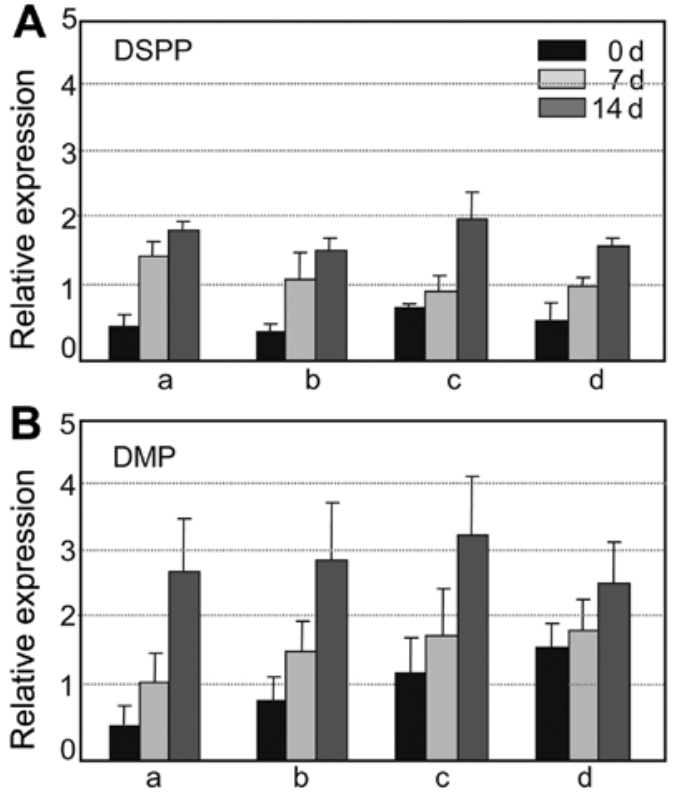

Figure 5. Dentinogenic differentiation potential of human dental pulp cells (hDPCs) following indirect co-culture with periodontal ligament stem cells (hPDLSCs) and/or gingival fibroblasts (hGFs). Following culture or co-culture for the indicated periods of time ( $0 \mathrm{~d}, 0$ days; $7 \mathrm{~d}, 7$ days; $14 \mathrm{~d}, 14$ days) with differentiation additives, the hDPCs were harvested for RNA preparation: black bars, without treatment with additives; light gray bars, 7-days of incubation with additives; dark gray bars, 14 days of incubation with additives. Transcript levels of the dentin markers, (A) DSPP and (B) DMP were estimated by RT-qPCR as described in the 'Materials and methods'. (A and B) Bar graph a, hDPCs cultured alone; bar graph b, co-culture of hDPCs with hGFs; bar graph c, coculture of DPCs with hPDLSCs; bar graph d, co-culture of hDPCs with both hGFs and hPDLSCs.

whether any soluble factors from these cells can provide a dentinogenic niche for dental pulp stem cells. The cell culture inserts were placed in wells seeded with hDPCs and the PDLSCs and/or hGFs were seeded within the inserts. This condition created a cell-cell barrier between the hDPCs and the hPDLSCs and/or hGFs, thus allowing the diffusion of medium and proteins. After 7 days of differentiation, the transcriptional expression of dentinogenic markers from the total RNA purified from the hDPCs was analyzed by RT-qPCR. The transcriptional expression of DSPP and DMP-1 increased when the cells were stimulated with the additives (Fig. 5). When the differentiation of the hDPCs was induced without co-culture, the expression of dentinogenic markers increased with the increasing duration of induction. The DSPP transcript level increased $\geq 3$-fold compared to the control (at 0 days of differentiation) after 7 and 14 days of incubation (Fig. 5A, bar graph a). The DMP-1 transcript level increased 2- and 5-fold compared to the control after 7 and 14 days of incubation, respectively (Fig. 5B, bar graph a). When the hDPCs were co-cultured with hGFs, the transcript levels of these markers also increased during differentiation to similar levels in comparison with those of the hDPCs cultured alone (Fig. 5A and $\mathrm{B}$, bar graph $\mathrm{b}$ ). When the hDPCs were co-cultured with hPDLSCs, the transcript levels of DSPP and DMP-1 were increased by $>2$-fold compared to the hDPCs cultured alone, even when the cells were not treated with differentiation additives (Fig. 5A and B, bar graphs a and c, black bars).
Although the expression levels of these genes were observed to be slightly increased after 7 and 14 days of differentiation in comparison with those of the hDPCs cultured alone, the rate of increment from the expression levels at day 0 was not significant under the co-culture conditions during differentiation (Fig. 5A and B, bar graph c, grey and dark grey bars). These data suggested that dentinogenic gene expression in hDPCs may be induced by indirect co-culture with hPDLSCs without inducing differentiation; however, the effects of indirect co-culture with hPDLSCs and those of treatment with differentiation additives on the mineralization of hDPCs were not synergistic. Additionally, when the hDPCs were co-cultured with hPDLSCs and hGFs in a co-culture insert, no significant synergistic effect was observed on the transcriptional expression of dentinogenic markers in hDPCs after 7 and 14 days of differentiation (Fig. 5A and B, graph d).

\section{Discussion}

Previous studies have investigated the differentiation potential of DPCs, PDLs and GFs isolated from the dental tissue of various species. Although it has been suggested PDLSCs, and GFs, as well as DPCs have stemness and differentiation potential $(17,21,42)$, systematic and comparative studies on the stemness of these oral cells are limited. In the present study, we confirmed the presence of a stem cell-like population in hDPCs, PDLSCs and hGFs comparatively by the systematic study of the osteo/dentinogenic differentiation of these cells. The hDPCs and hPDLSCs during early passage strongly expressed representative mesenchymal stem cell markers (Fig. 1A and B, panels b-d, f and g), whereas the already known dental apical papilla stem cell markers, CD24 and CD106, were not expressed in the hDPCs and hPDLSCs used in the present study (Fig. 1A and B, panels a and e). These findings strongly suggest that hDPCs and hPDLSCs are different lineages from dental apical papilla cells, although they contain a mesenchymal stem cell-like population $(21,24,26)$. Under osteogenic conditions, the hPDLSCs, as well as the hDPCs, highly mineralized and expressed dentinogenic markers (Figs. 3 and 4). During extended culture, the differentiation potential of the hPDLSCs peaked during early passage and decreased gradually after 8-9 passages (Fig. 4B, panels a and b), indicating that the hPDLSCs may have differentiated and/or lost their stemness potential during extended culture in a similar manner with the hDPCs, as desribed in a previous study (15). Moreover, we demonstrated that the hPDLSCs have dentinogenic potential (Fig. 4B). As previously reported, PDLSCs are also involved in the regeneration of the periodontal ligament, alveolar bone and cementum (21). These data indicate that hPDLSCs, as well as hDPCs may be a adult stem cell source for the regeneration of teeth and their periphery. It is disputed whether GFs contain stem cells that have the potential to regenerate multiple tissue cells. Mitrano et al (28) reported that primary cells from gingival connective tissue contain mesenchymal stem cells and have adipogenic, chondrogenic and osteogenic differentiation potential. In the present study, when hGFs from the attached gingival tissue were cultured, the cells from very early passages expressed the general mesenchymal stem cell markers, CD44, CD73, CD90, CD146 and CD166 [Fig. 1C and D (triangle in panels a-e)]. However, these cells did not show mineralization potential (Fig. 3), suggesting that there are few dentinogenic 
progenitors in primary cell populations. Previously, CarmonaRodriguez et al (42) reported that the mineralization potential of hGFs can be controversial, due to variations in the external environment, such as the composition of extracellular matrix and the existence of functional osteoblasts. In addition, the association, if any exits, between the expression of mesenchymal stem cell markers and dentinogenic potential remains to be elucidated. Indeed, although CD44, CD73 and CD90 were strongly expressed on the cell surface of the hGFs in our immunotyping experiment (Fig. 1C, panels b-d), dentinogenic markers, such as DSPP and DMP-1, were not expressed during differentiation (Fig. 4C, panel b). These results also indicate that mesenchymal stem cell markers, such as CD44 and CD90, are not proper markers for discriminating dentinogenic progenitors, and that more specific surface markers are required to detect dentinogenic stem/progenitor cells.

The stem cell niche refers to an in vivo or in vitro stem cell microenvironment that interacts with stem cells to regulate cell fate. In adult stem cells, stem cell niches maintain stem cells in a quiescent state; however, following tissue injury, the surrounding microenvironment signals stem cells to either promote self renewal or differentiation for tissue regeneration. Cell-cell interactions between stem cells, as well as extracellular matrix components, growth factors, cytokines and the physiochemical nature of the environment, including $\mathrm{pH}, \mathrm{Ca}^{2+}$ and metabolites, such as adenosine triphosphate (ATP), are important factors regulating stem cell characteristics within the niche (43). In this study, to investigate whether hPDLSCs and/or hGFs provide a dentinogenic niche of dental pulp stem cells during the developmental stage, we performed indirect co-culture experiments. Using culture inserts, soluble factors from the cultures of hPDLSCs and hGFs diffused into the hDPC culture at the bottom of the well. After 7 and 14 days of co-culture under differentiation conditions, the transcript levels of DSPP and DMP-1 did not increase in comparison with those in the hDPCs cultured alone (Fig. 5). In future studies, we aim to perform a co-culture experiment in order to investigate the direct effect on dentinogenesis by cell-tocell interaction, which would address the issue of whether a microenvironment from the extracellular matrix of hPDLSCs and/or hGFs can be a dentinogenic niche for the differentiation of hDPCs in vitro.

\section{Acknowledgements}

The present study was supported by the Priority Research Center Program (2013-0093829) funded by the National Research Foundation of Korea, and by the research fund of Dankook University (BK21 PLUS) in 2013.

\section{References}

1. Reynolds SD, Giangreco A, Power JH and Stripp BR: Neuroepithelial bodies of pulmonary airways serve as a reservoir of progenitor cells capable of epithelial regeneration. Am J Pathol 156: 269-278, 2000.

2. LaBarge MA and Blau HM: Biological progression from adult bone marrow to mononucleate muscle stem cell to multinucleate muscle fiber in response to injury. Cell 111: 589-601, 2002.

3. Morshead CM, Craig CG and van der Kooy D: In vivo clonal analyses reveal the properties of endogenous neural stem cell proliferation in the adult mammalian forebrain. Development 125 : 2251-2261, 1998
4. Otsuka H, Kusumi T, Kanai S, Koyama M, Kuno Y and Takizawa R: Stem cell factor mRNA expression and production in human nasal epithelial cells: contribution to the accumulation of mast cells in the nasal epithelium of allergy. J Allergy Clin Immunol 102: 757-764, 1998.

5. Seigel GM, Sun W, Salvi R, Campbell LM, Sullivan S and Reidy JJ: Human corneal stem cells display functional neuronal properties. Mol Vis 9: 159-163, 2003.

6. Lemischka IR, Raulet DH and Mulligan RC: Developmental potential and dynamic behavior of hematopoietic stem cells. Cell 45: 917-927, 1986

7. Wang J, Wang X, Sun Z, Yang H, Shi S and Wang S: Stem cells from human-exfoliated deciduous teeth can differentiate into dopaminergic neuron-like cells. Stem Cells Dev 19: 1375-1383, 2010.

8. Miura M, Gronthos S, Zhao M, Lu B, Fisher LW, Robey PG and Shi S: SHED: stem cells from human exfoliated deciduous teeth. Proc Natl Acad Sci USA 100: 5807-5812, 2003.

9. Gronthos S, Mankani M, Brahim J, Robey PG and Shi S: Postnatal human dental pulp stem cells (DPSCs) in vitro and in vivo. Proc Natl Acad Sci USA 97: 13625-13630, 2000.

10. Arthur A, Rychkov G, Shi S, Koblar SA and Gronthos S: Adult human dental pulp stem cells differentiate toward functionally active neurons under appropriate environmental cues. Stem Cells 26: 1787-1795, 2008.

11. Gronthos S, Brahim J, Li W, et al: Stem cell properties of human dental pulp stem cells. J Dent Res 81: 531-535, 2002.

12. Shi S, Robey PG and Gronthos S: Comparison of human dental pulp and bone marrow stromal stem cells by cDNA microarray analysis. Bone 29: 532-539, 2001.

13. Tziafas D: Basic mechanisms of cytodifferentiation and dentinogenesis during dental pulp repair. Int J Dev Biol 39: 281-290, 1995.

14. Kuznetsov SA, Krebsbach PH, Satomura K, Kerr J, Riminucci M, Benayahu D and Robey PG: Single-colony derived strains of human marrow stromal fibroblasts form bone after transplantation in vivo. J Bone Miner Res 12: 1335-1347, 1997.

15. Min JH, Ko SY, Cho YB, Ryu CJ and Jang YJ: Dentinogenic potential of human adult dental pulp cells during the extended primary culture. Hum Cell 24: 43-50, 2011.

16. Shi S and Gronthos S: Perivascular niche of postnatal mesenchymal stem cells in human bone marrow and dental pulp. J Bone Miner Res 18: 696-704, 2003.

17. Mensing N, Gasse H, Hambruch N, Haeger JD, Pfarrer C and Staszyk C: Isolation and characterization of multipotent mesenchymal stromal cells from the gingiva and the periodontal ligament of the horse. BMC Vet Res 7: 42, 2011.

18. Hayashi Y, Imai M, Goto Y and Murakami N: Pathological mineralization in a serially passaged cell line from rat pulp. J Oral Pathol Med 22: 175-179, 1993.

19. Iwata T, Yamakoshi Y, Simmer JP, Ishikawa I and $\mathrm{Hu}$ JC: Establishment of porcine pulp-derived cell lines and expression of recombinant dentin sialoprotein and recombinant dentin matrix protein-1. Eur J Oral Sci 115: 48-56, 2007.

20. Nakashima M: Establishment of primary cultures of pulp cells from bovine permanent incisors. Arch Oral Biol 36: 655-663, 1991.

21. Seo BM, Miura M, Gronthos S, et al: Investigation of multipotent postnatal stem cells from human periodontal ligament. Lancet 364: 149-155, 2004.

22. Ivanovski S, Gronthos S, Shi S and Bartold PM: Stem cells in the periodontal ligament. Oral Dis 12: 358-363, 2006.

23. Liu Y, Zheng Y, Ding G, et al: Periodontal ligament stem cell-mediated treatment for periodontitis in miniature swine. Stem Cells 26: 1065-1073, 2008.

24. Mrozik K, Gronthos S, Shi S and Bartold PM: A method to isolate, purify, and characterize human periodontal ligament stem cells. Methods Mol Biol 666: 269-284, 2010.

25. Takada H, Mihara J, Morisaki I and Hamada S: Induction of interleukin-1 and -6 in human gingival fibroblast cultures stimulated with Bacteroides lipopolysaccharides. Infect Immun 59: 295-301, 1991.

26. Gagari E, Rand MK, Tayari L, Vastardis H, Sharma P, Hauschka PV and Damoulis PD: Expression of stem cell factor and its receptor, c-kit, in human oral mesenchymal cells. Eur J Oral Sci 114: 409-415, 2006.

27. Okazaki M, Yoshimura K, Uchida G and Harii K: Elevated expression of hepatocyte and keratinocyte growth factor in cultured buccal-mucosa-derived fibroblasts compared with normal-skinderived fibroblasts. J Dermatol Sci 30: 108-115, 2002. 
28. Mitrano TI, Grob MS, Carrion F, et al: Culture and characterization of mesenchymal stem cells from human gingival tissue. J Periodontol 81: 917-925, 2010.

29. Covas DT, Panepucci RA, Fontes AM, et al: Multipotent mesenchymal stromal cells obtained from diverse human tissues share functional properties and gene-expression profile with $\mathrm{CD} 146^{+}$ perivascular cells and fibroblasts. Exp Hematol 36: 642-654, 2008.

30. Majeti R, Park CY and Weissman IL: Identification of a hierarchy of multipotent hematopoietic progenitors in human cord blood. Cell Stem Cell 1: 635-645, 2007.

31. Zhu H, Mitsuhashi N, Klein A, et al: The role of the hyaluronan receptor CD44 in mesenchymal stem cell migration in the extracellular matrix. Stem Cells 24: 928-935, 2006.

32. Dennis JE, Carbillet JP, Caplan AI and Charbord P: The STRO $-1^{+}$marrow cell population is multipotential. Cells Tissues Organs 170: 73-82, 2002.

33. Barry F, Boynton R, Murphy M, Haynesworth S and Zaia J: The SH-3 and SH-4 antibodies recognize distinct epitopes on CD73 from human mesenchymal stem cells. Biochem Biophys Res Commun 289: 519-524, 2001.

34. Craig W, Kay R, Cutler RL and Lansdorp PM: Expression of Thy-1 on human hematopoietic progenitor cells. J Exp Med 177: 1331-1341, 1993.

35. Simmons PJ and Torok-Storb B: Identification of stromal cell precursors in human bone marrow by a novel monoclonal antibody, STRO-1. Blood 78: 55-62, 1991.

36. Russell KC, Phinney DG, Lacey MR, Barrilleaux BL, Meyertholen KE and O'Connor KC: In vitro high-capacity assay to quantify the clonal heterogeneity in trilineage potential of mesenchymal stem cells reveals a complex hierarchy of lineage commitment. Stem Cells 28: 788-798, 2010.
37. Barreiro O, Yanez-Mo M, Serrador JM, et al: Dynamic interaction of VCAM-1 and ICAM-1 with moesin and ezrin in a novel endothelial docking structure for adherent leukocytes. J Cell Biol 157: 1233-1245, 2002.

38. Majumdar MK, Banks V, Peluso DP and Morris EA: Isolation, characterization, and chondrogenic potential of human bone marrow-derived multipotential stromal cells. J Cell Physiol 185: 98-106, 2000

39. Morsczeck C, Schmalz G, Reichert TE, Vollner F, Galler K and Driemel O: Somatic stem cells for regenerative dentistry. Clin Oral Investig 12: 113-118, 2008.

40. Binulal NS, Deepthy M, Selvamurugan N, et al: Role of nanofibrous poly(caprolactone) scaffolds in human mesenchymal stem cell attachment and spreading for in vitro bone tissue engineering-response to osteogenic regulators. Tissue Eng Part A 16: 393-404, 2010.

41. Yang X, Yang F, Walboomers XF, Bian Z, Fan M and Jansen JA: The performance of dental pulp stem cells on nanofibrous PCL/gelatin/nHA scaffolds. J Biomed Mater Res Part A 93: 247-257, 2010.

42. Carmona-Rodriguez B, Alvarez-Perez MA, Narayanan AS, et al: Human Cementum Protein 1 induces expression of bone and cementum proteins by human gingival fibroblasts. Biochem Biophys Res Commun 358: 763-769, 2007.

43. Li L and Xie T: Stem cell niche: structure and function. Annu Rev Cell Dev Biol 21: 605-613, 2005. 